\title{
STUDY OF VARIOUS ENERGY EFFICIENT PROTOCOL IN WIRELESS SENSOR NETWORK
}

\author{
Kawaljit Kaur $^{1} \&$ Amarpreet Singh ${ }^{2}$
}

Abstract-WSN (Wireless Sensor Network) is a communication network that is comprised of various tiny sensor nodes and these sensor nodes operate on the basis of the energy or battery powers that are allotted to them initially. These sensor nodes are independent of each other. The process of communication in the WSN consumes high amount of energy since sensor nodes consumes transmitting energy and $\mathrm{CH}$ (Cluster Head) nodes consumes receiving as well as transmitting energy. But it is a fact that if the amount of energy consumption goes high consequently the lifetime of the network goes down. To reduce the energy consumption is one of the tedious tasks to perform in WSN. There are various techniques that are specifically developed to create an energy efficient network. Various optimization techniques can also applied along with these energy efficient protocols to enhance their performance. This study represents the various energy efficient protocols and optimization techniques.

Keywords-Wireless Sensor Network, Energy Efficient, Optimization techniques

\section{INTRODUCTION}

Wireless sensor networks are more than a decade back and plentiful. Due to this fact, it has several areas where different researchers have been working. They have been digging out the area and focusing on its several aspects. These networks are equipped with computing, sensing as well as wireless communication capabilities. Additionally, WSN consists of several sensor nodes which are smaller in size and less costly that deployed in different environments in order to form a special sensor network. Consequently, network may have tens, hundreds or even thousands of sensors that are formed to achieve different domains such as fault tolerant, reliability, accuracy and efficiency in sensor network covering a wider region [1].

Therefore while deploying a sensor network it is mandatory to define the coverage area of the network in such a way that it can become efficient and effective to deploy the senor nodes in the network so that the maximum coverage can be obtained and the connectivity of the nodes can also become easy. Three nodes namely management node, single sensor node and sink node are required to create a wireless sensor node deployment for roads. The workings of these three nodes are as follows [4]:

- Management Node: Firstly data is collected from internet, stored in database and then records are maintained to provide the search services.

- Sink Node: Information regarding road, people and traffic flow received by sink node, and it sends the results to the management node.

- Single Sensor Node: It comprises of vehicle and fixed nodes. Fixed nodes are buried in the road and on both sides of road equally to check the status of road and also collect data related to traffic on the road.

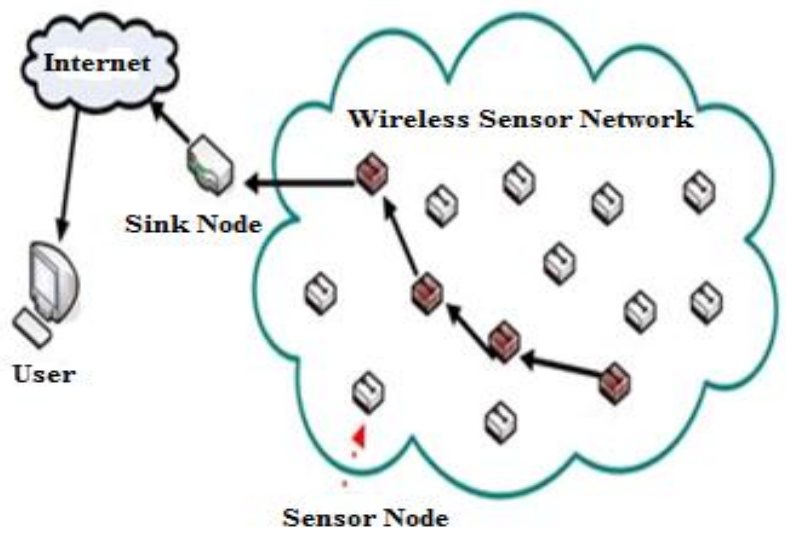

Figure 1: Wireless Sensor Network [5]

\footnotetext{
${ }^{1}$ M.tech Research Scholar, ACET Amritsar, IKG PTU Jalandhar

${ }^{2}$ Associate Professor, ACET Amritsar, IKG PTU Jalandhar
} 
In the above figure 1, a wireless sensor network has defined where the main components consisted in the networks are sensing unit, transmission unit, processing unit and power unit.

Sensor nodes used in the environment of WSN are mostly battery powered and deployed in the least human supervision such as battlefields, forests etc. like environment. So, replacement or recharging of these batteries is not practical feasible. To avoid this factor of disruption while service, two approaches have been followed. In the first approach, abundance of sensor nodes are deployed which provides redundancy in the data reported by sensor nodes. Therefore, if any of the sensor node runs out of battery, similar nodes are covering for the same. Moreover, serious issues are mitigating. In the second approach of WSN, any algorithm can be used to pose any processing burden. The early phases of development, designing and deployment of Wireless sensor networks are focused on the conservations of the power resources of the sensor nodes. There are several studies performed in optimizing the power consumption that provided high efficiency energy consumption mechanisms to continue the operation while having enough energy.

These abilities of WSN makes them capable in standing out as a platform where several applications like power systems, transportation, healthcare, air polluting monitoring and roadside applications can be deployed.

On the counterpart, WSNs have been suffering from several limitations in their capabilities like small memory, low processing power and limited energy. Sensor nodes in the network consumes high amount of energy while sensing data processing and communicating without any support of constant power supply with the difficulty of replacement. Among these tasks, communication employs high amount of energy and consequently WSN expires by the time battery runs out of energy [6]. The expiration affects performance, quality as well as lifetime of the network.

Therefore, in critical applications, lifetime of the network is decisive because human lives are important to be secure. So, strict energy constraints are equally important similar to lifetime of the sensor networks and reduced energy consumption while designing WSN.

\section{CLUSTERING PROTOCOLS}

If each and every senor node present in the network starts communicating through base station or sends its data directly to the base station in that case, more energy will be consumed because of the long distances to the base station. Moreover, collision and congestion in the network will also consume high amount of energy as data needs to be retransmitted again and again. In order to solve these concerns, a clustering mechanism for WSN has introduced. In such mechanism, nodes in the defined cluster will send its data to their respective cluster heads rather than base station. This process will shorten the total distance covered by the nodes and hence, the energy will be less consumed. These clusters heads forward this data to the base station [7]. Additionally, process of aggregating as well as fusing of data at the cluster head in the cluster provided help in enucleating data redundancy.

The clustering scheme comprised of two types of nodes first is CM (cluster members) and second is CHs. The CMs sense the data from its surroundings periodically and then this sensed data is transmitted to the CHs. Then the CHs perform data aggregation on received data and forward it to the base station (BS) [5]. This communication can be done in two different manners. One way is the single hop communication and other is multi-hop communication. In multi-hop communication, the $\mathrm{CHs}$ consume energy for receiving the data, for aggregating the data and to transmit the data packets to its adjacent cluster heads (i.e. Inter-Clusters). The utilization of $\mathrm{CHs}$ protocols aim to minimize the energy consumption whereas, in single hop communication the $\mathrm{CHs}$ directly send the data packets to the sink node [27]. In this process there are high chances of energy consumption.

Being a cluster head node of the cluster, it consumes high amount of energy in comparison with other general nodes or cluster members but the solution of this problem is also available where other nodes are also selected as a cluster head rotationally. Several protocols have been proposed which helps in reducing consumption of energy as well as increasing network lifetime. The clustering protocols are as follows [8]:

1. Low-Energy Adaptive Clustering Hierarchy (LEACH)

2. Power efficient gathering in sensor information systems (PEGASIS)

3. Hybrid energy efficient distributed clustering (HEED)

4. Energy Efficient Unequal Clustering (EEUC)

5. Fast Local Clustering Service (FLOC)

These clustering protocols can be combined with the optimization algorithm to optimize the energy consumption of sensor nodes. Some of the optimization algorithms have described in below section which can be collaborated with the clustering protocols.

\section{OPTIMIZATION ALGORITHM}

This section of the paper describes several optimization algorithms which highlights their merits and demerits along with noble features. The algorithms involved are Particle Swarm Optimization (PSO) algorithm, Ant Colony Optimization (ACO) algorithm, Artificial Bee Colony ( $\mathrm{ABC}$ ) algorithm, Bacterial Colony Optimization (BCO) algorithm, Artificial Immune System (AIS), Gray Wolf Optimizer (GWO) algorithm, Bat algorithm (BA), Bees Algorithm [9]. 
3.1 Particle Swarm Optimization

Particle Swarm Optimization (PSO) is a global optimization algorithm to deal with problems that can represent an optimal solution as a point or surface in n dimensional space proposed by Kennedy and Eberhart in 1995. Hypotheses are plotted in this space and seeded at initial velocity as well as communication channels between particles. The particles move through the solution space and are evaluated according to some criteria after each time step. Over time, the particles are accelerated towards particles with better fitness values within the communication group. The formula used for the evaluation is as follows:

$v_{i d}^{t+1}=v_{i d}^{t}+c_{1} \cdot \operatorname{rand}(0,1) \cdot\left(p_{i d}^{\mathrm{t}}-x_{i d}^{\mathrm{t}}\right)+c_{2} \cdot \operatorname{rand}(0,1) \cdot\left(p_{g d}^{\mathrm{t}}-x_{i d}^{\mathrm{t}}\right)$

$x_{i d}^{t+1}=x_{i d}^{t}+w_{i d}^{t+1}$

In the equation 1 and $2, v_{i d}^{t}$ and $x_{\text {Id }}^{t}$ are considered as a velocity and position of particle respectively. Where, $\mathrm{d}$ is dimension in search space, $i$ is particle index and iteration number is represented through $t . c_{1}$ and $c_{2}$ represent the speed which means the length of the flying toward the optimal particles. Rand $(0,1)$ shows the random number generated between 0 and 1 . The exploration and exploitation happens based upon these bales. If difference between particle's best i.e. $p_{i d}^{t}$ and previous position of particle i.e. $x_{i d}^{t}$ and best population of all time i.e. $p_{g d d}^{t}$ and $x_{i d}^{t}$ i.e. pervious particle's position are large. On the other side, exploitation occurs if both of the values are small.

\subsection{Ant Colony Optimization}

Ant Colony Optimization (ACO) is a kind of optimization algorithm modeled on the behavior of an ant colony proposed by Marco Dorigo in the year of 1992 at the time of his PhD thesis. ACO is a useful probabilistic approach to dealing with finding better routes through graphs. The artificial "ants" simulation agent identifies the best solution by moving the parameter space representing all possible solutions. Natural ants lay pheromones that guide each other to resources while exploring the environment. As simulated "ant" has also recorded the location and quality of the solution, more ants in a later simulation can find a better solution. In order to find the best solution starting from choosing the next node with the help of following equation:

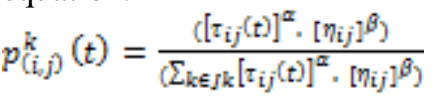

In the equation $3, \mathrm{p}_{\mathrm{i}, \mathrm{j}}$ is the probability of going from i node to node $\mathrm{j} . \mathrm{J}_{\mathrm{k}}$ are those nodes when an ant allowed them to travel from node $\mathrm{i}$. In the visibility between node I and node $\mathrm{j}, \mathrm{n}_{\mathrm{ij}}$ contributes in it. $\tau_{\mathrm{i} j}(t)$ shows un-evaporated pheromone amount between node $i$ and $j$ at the time interval t. Its influence is controlled by $\alpha$ and $\beta$ in the equation 3. If $\alpha$ is greater or higher in that case searching behavior of ant depends upon the pheromone and on the counterpart if $\beta$ is higher than searching behavior of ant based upon the visibility or knowledge.

\subsubsection{Artificial Bee Colony Algorithm}

This algorithm was initially proposed by Dervis Karaboga in the year of 2005. It has three stages of Adopted Bee, Spectator bee, Scout Bee. At the adopted bee and spectator bee stage, the bees utilize the source by local search near selected solutions based on deterministic selection. At the adopted bee stage, and the viewer's bee stage, we make stochastic choices. In the Scout Bee phased, analogy of abandoning tired sources of food in the search process, solutions that are not beneficial to the progress of the search are abandoned and new solutions are inserted to explore new areas within the search space. This algorithm has well-balanced exploration and exploitation capabilities [23].

\subsubsection{Bacterial Colony Optimization}

This algorithm is based on a life cycle model simulating the typical behavior of E. Cole during their entire life cycle, including chemical taxis, communication, exclusion, replication and migration. The Eshel Ben-Jacob research group at the University of Tel Aviv developed a fractal model of bacterial colonies and identified the linguistic and social patterns in the colonial life cycle in the context of network theory Transmission and self organization are studied, microbial intelligence and cooperation of microorganisms [24].

\subsubsection{Artificial Immune Systems}

The artificial immune system (AIS) is to use the abstract structure and function of the immune system for the calculation system. To study the application of these systems to solve calculation problems from mathematics, engineering, information technology.AIS is a subfield of biologically influenced computing and natural computation interested in machine learning and belongs to the broad field of artificial intelligence [26].

\subsubsection{Gray Wolf Optimizer}

The Gray wolf optimizer (GWO) algorithm imitates the leadership hierarchy and hunting mechanism of gray wolves in nature. Four kinds of gray wolves such as alpha, beta, delta and omega are adopted to simulate the leadership hierarchy. In addition, three major steps are implemented to carry out optimization: hunting, exploring prey, enclosing, and attacking [10]. 


\subsubsection{Bat Algorithm}

The bat algorithm (BA) is a group intelligence based algorithm, inspired by the echolocation behaviour of micro bats. BA uses frequency tuning and automatic balancing of exploration and utilization by controlling loudness and pulse release rate [10].

\subsubsection{The Bees Algorithm}

This algorithm, modeled based on hunting behaviors of bees, combines global exploratory search with local exploratory search. While a small number of artificial bees (scouts) randomly searches for solution spaces (environments) to find solutions to high fitness (highly profitable food sources), the majority of the population is fitness optimal solutions. A definitive recruitment procedure to simulate biological bee's flapping dance is to inform the owner of the scouting survey results and to distribute the feed according to the neighborhood fitness chosen for local exploration used. When the search in the vicinity of the solution stagnates, the local optimum is found and the site is abandoned. In summary, the Bees algorithm searches for the most promising areas of the solution space at the same time, and searches for new advantageous areas and continuously sample them [25].

\section{RELATED WORK}

There are number of models that have been performed by several researchers that attempted to optimize several parameters such as end-to-end latency, reliability and energy efficiency. In the past, most of the focus was on minimizing the energy consumption as well as on the latency. Certain applications in WSN such as device to device communication, smart grid monitoring and industrial monitoring applications require high reliability along with strict real time requirements. Some of the work of researchers in WSN clustering protocols have described below.

Hongwei Chen et al (2013) [19] defined that LEACH is most prominent energy efficient mechanism that is widely used for enhancing the performance of the WSN. LEACH performs the random selection. LEACH did not assure the optimization and number of $\mathrm{CH}$. Therefore in order to perform optimization of the $\mathrm{LEACH}$, this study presents the optimal energy efficient $\mathrm{CH}$ selection and was named as LEACH-G. This mechanism initially elected the optimum number of $\mathrm{CH}$ on the basis of energy model of LEACH protocol and then implemented the LEACH-G for selection of $\mathrm{CH}$ from list of optimum CHs. After that author also compared the performance of LEACH-G and LEACH on NS2 simulation. After calculating the optimum number of CHs, the energy is evenly dispersed to the network. The simulation results proved the efficiency of LEACH-G over traditional LEACH with respect to the lifetime of the network and the energy consumption of the nodes in the network.

V Loscrì (2005) [20] represented that WSN is applied to various domains. The author proposed an energy efficient mechanism for WSN which utilized the more prominent method i.e. LEACH. This was designed for sensor network so that the surrounding can be monitored remotely. In this case the data packets from the nodes were transmitted to the sink node and this might be possible that the sink can be located far away from the nodes where the end user is monitoring the network and have accessibility to the data. The proposed work developed the two-level hierarchical protocol i.e. TL-LEACH to save the energy of the network. In this protocol the primary and secondary $\mathrm{CH}$ rotate randomly and allot the equal amount of energy in the network specifically in for high density networks. It localized the coordination to obtain scalable and robust network. The proposed mechanism was simulated by using NS2 and observed the proposal as an efficient mechanism.

Sharma Nishi (2013) [21] defined that the WSN is a collection of tiny sensors which operates on the basis of the battery power and has limited space for storing the information. These small battery operated sensor nodes sense the data from its geographical surroundings and then this information is transferred to the base station in the form of data packets. The real life applications work on the basis of heterogeneity. In this study, author performed a research on a protocol with energetic heterogeneity to evaluate the LEACH. The objective of this study was to examine the LEACH which is a homogeneous network protocol and analysed how the energy level of the sensors get affected with the heterogeneity. MATLAB software was preferred to simulate the proposed work and it was presented that the proposed discrete heterogeneity protocol was capable to decrease the energy consumption of the nodes and also results to the enhancement in the lifetime of the network.

Liu Jenn-Long (2013) [22] developed genetic algorithm based on adaptive clustering protocol. The proposed system utilized the concept of optimal probability prediction with respect to the lifetime of the WSN. The working of the LEACH-GA was defined in the terms of preparation, setup, and steady state phase. At the time of the preparation phase, initially the $\mathrm{CH}$ selection is performed and then the status node identities and location of the nodes is stored. After receiving the data packets from the sensor the sink node explored the GA to find out the optimal probability of the $\mathrm{CH}$ by reducing the level of energy consumption that was needed for the completion of the first iteration. Then the sink node broadcast a message in the network which depicted the optimal probability value of all the sensor nodes and then created the clusters on the basis of the evaluated probability values. The preparation phase executes only for once while initialization of the network only. The rest of the phases had similar working process as LEACH. Simulation was performed to show that the proposed technique effectively produced optimal energy consumption of WSN which automatically extends the lifetime of the network.

Bakaraniya Paul (2013) [23] addressed that the energy saving is one of the most unsolved issue in wireless sensor networks. This research work was conducted by author to develop the modified protocol Kmedoids-LEACH (K-LEACH) with an objective to increase the existence time of the network. This was achieved by the balancing the level of energy consumption of the network. The kmedoids clustering algorithm was applied for homogeneous clustering and then $\mathrm{CH}$ selection was performed by using collaboration of two techniques i.e. Euclidean distance and maximum residual energy. 


\section{CONCLUSION}

To develop an energy efficient wireless sensor network is a challenging issue. Various energy efficient protocols have been developed in the past and various authors utilized these protocols for their research work but still a satisfactory level of the energy efficiency in WSN was not achieved. After then as per related work of this study it has been seen that various researches had been conducted by using optimization algorithms along with energy efficient protocols. On the basis of the related work it is concluded that in future fuzzy inference system can be combined with genetic algorithm for evaluating the fitness function in order to elect $\mathrm{CH}$ in WSN.

\section{REFERENCES}

[1] Er. Rimpy Sharma et al, "Evaluating The Performance Of Density Grid-Based Clustering Using Abc Technique For Efficient Routing In WsNs",2017 51st Annual Conference on Information Sciences and Systems (CISS), pp. 1 - 7, 2017

[2] Monica R Mundada et al, "A Study On Energy Efficient Routing Protocols In Wireless Sensor Networks", International Journal of Distributed and Parallel Systems (IJDPS), vol. 3, no. 3, pp. 311-330, May 2012

[3] Arun L. Kakhandki et al, “An Efficient Transceiver Optimization based Routing Technique for Wireless Sensor Network”, 2016 International Conference on Communication and Electronics Systems (ICCES), pp. 1 - 5, 2016

[4] Nikolaos A. Pantazis et al, "Energy-Efficient Routing Protocols in Wireless Sensor Networks: A Survey", IEEE Communications Surveys \& Tutorials, vol. 15, no. 2, pp. 551-591, 2013

[5] Pankaj Chauhan and Tarun Kumar, "Power Optimization in Wireless Sensor Network: A Perspective", International Journal of Engineering and Technical Research, Vol. 3, No. 5, Pp. 273-277, May 2015

[6] Swati Shamkuwar et al, "A Review on Energy Efficient Routing Protocols in Wireless Sensor Networks", International Journal of Emerging Technology and Advanced Engineering, vol. 4, no. 3, pp. 653-657, March 2014

[7] Ruchi Aggarwal et al, "Various Optimization techniques used in Wireless Sensor Networks", International Research Journal of Engineering and Technology (IRJET), vol. 3, no. 6, pp. 2085-2090, June 2016

[8] Mohammad Baniata and Jiman Hong, "Energy-Efficient Unequal Chain Length Clustering for Wireless Sensor Networks in Smart Cities", Wireless Communications and Mobile Computing, Vol. 2017, Pp. 1-12, 2017

[9] Mohd Nadhir Ab Wahab et al, “A Comprehensive Review of Swarm Optimization Algorithms”, PLoS ONE, Vol. 10, No. 5, Pp. 1-36, May 2015

[10] Loveneet Kaur et al, “Optimization techniques for Routing in Wireless Sensor Network", (IJCSIT) International Journal of Computer Science and Information Technologies, vol. 5, pp. 4719-4721, 2014

[11] Zesong Fei et al, "A Survey of Multi-Objective Optimization in Wireless Sensor Networks: Metrics, Algorithms and Open Problems", IEEE Communications Surveys \& Tutorials, vol. 19, no. 1, pp. 550-586, 2017

[12] Rong Du et al, "Lifetime Maximization for Sensor Networks with Wireless Energy Transfer", 2016 IEEE International Conference on Communications (ICC), pp. $1-6,2016$

[13] Anju et al, "Energy Efficient Clustering In Wireless Sensor Network: A Review", Pp. 12-14

[14] Shilpa Mahajan et al, "Clustering in Wireless Sensor Networks: A Review”, International Journal of Advanced Research in Computer Science, Vol. 7, No. 3, Pp. 198-201, June 2016

[15] Shantala Devi Patil et al, "Clustering in Mobile Wireless Sensor Networks: A Review", International Journal of Advanced Networking \& Applications (IJANA), Pp. 133-136, 2016

[16] Praveen Lalwani et al, "Bacterial Foraging Optimization Algorithm for CH selection and routing in wireless sensor networks", Recent Advances in Information Technology (RAIT), 2016 3rd International Conference on, March 2016

[17] Wendi Rabiner Heinzelman et al "Energy-Efficient Communication Protocol for Wireless Microsensor Networks", IEEE, pp 1-10, 2000

[18] Mundada Monica R et al, “ a study on energy efficient routing protocols in wireless sensor networks”, IJDPS, pp 311-330, Vol 3, No.3, 2012

[19] Hongwei Chen, “LEACH-G: an Optimal Cluster-heads Selection Algorithm based on LEACH”, Journal of software, pp 2661-2667, Vol- 8, 2013

[20] V. Loscrì , “A Two-Level Hierarchy for Low-Energy Adaptive Clustering Hierarchy (TL-LEACH)” IEEE, 2005

[21] Sharma Nishi et al , "Heterogeneous LEACH Protocol for Wireless Sensor Networks", Int. .J. Advanced Networking and Applications, pp 1825-1829, Vol: 5, Issue : 01, 2013

[22] Liu Jenn-Long et al, “ LEACH-GA: Genetic Algorithm-Based Energy-Efficient Adaptive Clustering Protocol for Wireless Sensor Networks” IEEE, pp 79-85 Vol - 1, No- 1, 2011

[23] Bakaraniya Parul et al, “ K-LEACH: An improved LEACH Protocol for Lifetime Improvement in WSN”, IJETT, pp 1521-1526 Vol-4, Issue-5, 2013

[24] Wei Fang et al , “A Review of Quantum-behaved Particle Swarm Optimization”.2014

[25] Teodorović Dušan et al, “Swarm intelligence systems for transportation engineering: Principles and applications”, ELSEVLER, pp 651-667, 2008

[26] Feng-Cheng Chang et al , “A refactoring method for cache-efficient swarm intelligence algorithms” ELSEVIER, Vol 192, pp 39-49, 2012

[27] Ramandeep Kaur, Er. Amarpreet Singh "A review on energy efficient clustering protocols based on LEACH in wireless sensor network" Australian Journal of Information Technology and Communication, Volume II Issue III September 2015 ISSN 2203-2843

[28] Ramandeep kaur,Er Amarpreet Singh "Weighted Clustering Algorithm based on O-Leach (WC-OLEACH) in Wireless Sensor Network" International Journal of Innovations in Engineering and Technology (IJIET), Volume 6 Issue 3 February 2016 